\title{
Recipients with In Utero Induction of Tolerance Upregulated MHC Class I in the Engrafted Donor Skin
}

\author{
Jeng-Chang Chen, ${ }^{1}$ Liang-Shiou Ou, ${ }^{2}$ Hsiu-Yueh Yu, ${ }^{3}$ Ming-Ling Kuo, ${ }^{4}$ \\ Pei-Yeh Chang, ${ }^{1}$ and Hsueh-Ling Chang ${ }^{3}$ \\ ${ }^{1}$ Department of Surgery, Chang Gung Children's Hospital, College of Medicine, Chang Gung University, 5 Fu-Shin Street, \\ Kweishan, Taoyuan 333, Taiwan \\ ${ }^{2}$ Department of Allergy, Asthma and Rheumatology, Chang Gung Children's Hospital, College of Medicine, \\ Chang Gung University, 5 Fu-Shin Street, Kweishan, Taoyuan 333, Taiwan \\ ${ }^{3}$ Pediatric Research Center, Chang Gung Children's Hospital, 5 Fu-Shin Street, Kweishan, Taoyuan 333, Taiwan \\ ${ }^{4}$ Department of Microbiology and Immunology, Graduate Institute of Biomedical Sciences, College of Medicine, Chang Gung University, \\ 259 Wen-Hwa 1st Road, Kweishan, Taoyuan 333, Taiwan
}

Correspondence should be addressed to Jeng-Chang Chen; jch.chen@msa.hinet.net

Received 23 April 2014; Revised 23 June 2014; Accepted 23 June 2014; Published 20 July 2014

Academic Editor: Giuseppe Murdaca

Copyright (C) 2014 Jeng-Chang Chen et al. This is an open access article distributed under the Creative Commons Attribution License, which permits unrestricted use, distribution, and reproduction in any medium, provided the original work is properly cited.

\begin{abstract}
The alterations in MHC class I expression play a crucial step in immune evasion of cancer or virus-infected cells. This study aimed to examine whether tolerized grafts modified MHC class I expression. FVB/N mice were rendered tolerant of C57BL/6 alloantigens by in utero transplantation of C57BL/6 marrows. Postnatally, engrafted donor skins and leukocytes were examined for their MHC expression by quantitative real-time PCR and flow cytometry. Engrafted donor skins upregulated their MHC class I related gene transcripts after short-term (1 2 weeks) or long-term ( $>1$ month) engraftment. This biological phenomenon was simultaneously associated with upregulation of TAP1 gene transcripts, suggesting an important role of TAP1 in the regulation of MHC class I pathway. The surface MHC class I molecules of $\mathrm{H}-2 \mathrm{~K}^{\mathrm{b}}$ in engrafted donor leukocytes consistently showed overexpression. Conclusively, the induction of allograft tolerance involved biological modifications of donor transplants. The overexpression of MHC class I within engrafted transplants of tolerant mice might be used as the tolerance biomarkers for identifying a state of graft tolerance.
\end{abstract}

\section{Introduction}

Organ transplantation has been the standard practice for failed or failing organs. However, long-term graft survivals rely upon immunosuppressive therapies that lessen or prevent rejection episodes at the cost of direct organ toxicity, susceptibility to opportunistic infections, malignancy, and accelerated cardiovascular diseases $[1,2]$. Despite a barely imagined concept in the past, achieving tolerance to transplanted organs is the best way to solve the problems imposed by immunosuppressive therapies. Thus, modern transplantation medicine is moving away from the exploration of novel immunosuppressive agents toward the induction of allograft tolerance in organ recipients. It causes a pressing need to search for unique tolerance biomarkers as surrogate endpoints of immunosuppressive strategies, or measures for predicting successful tolerance induction $[2,3]$. Recipients with graft tolerance retain well-functioning grafts after cessation of immunosuppressive regimens, referred to as operational tolerance [4]. They are the best proof of concept for immunosuppressive-free graft survivals and the potential candidates for the quest of tolerance signatures $[1,5-7]$. However, this unique paradigm of human tolerance develops exclusively under the aegis of antecedent immunosuppressive therapies. The effects of immunosuppressive cessation on these recipients remain a matter of concern to the interpretation of tolerance biomarkers generated $[1,8]$. Several lines of evidence have revealed a critical role for MHC class 
I (MHC-I) molecules in immune evasion of virus-infected or cancer cells $[9,10]$, suggesting that tolerized grafts might have to undergo similar biological alterations. In this tolerance model without the employment of immunosuppression, engrafted donor skins upregulated MHC-I associated gene transcripts and engrafted donor cells also overexpressed surface MHC-I molecules. Thus, graft tolerization facilitated MHC-I expression within donor grafts.

\section{Materials and Methods}

2.1. Mice. $\mathrm{FVB} / \mathrm{N}\left(\mathrm{H}-2^{\mathrm{q}}\right)$ mice were used as recipients, $\mathrm{C} 57 \mathrm{BL} / 6\left(\mathrm{H}-2^{\mathrm{b}}\right)$ as donors, and $\mathrm{C} 3 \mathrm{H}\left(\mathrm{H}-2^{\mathrm{k}}\right)$ as the source of third-party alloantigens. They were purchased at their age of 6-8 weeks and bred for this study with the approval of the Committee on Animal Research at Chang Gung Memorial Hospital. Recipient females were caged with males in the afternoon and checked for vaginal plugs the following morning. The day when the plug was observed was designated as day 0 of the pregnancy.

\subsection{Preparation of Donor Cells and In Utero Transplantation.} C57BL/6 bone marrow cells (BMCs) were harvested by flushing the tibias and femurs with phosphate buffer saline (PBS) using a 26-gauge needle, layered over NycoPrep 1.077A (Nycomed, Pharma AS, Oslo, Norway), and centrifuged at $600 \mathrm{~g}$ for 25 minutes. Light-density BMCs were then depleted of T-cells by anti-CD3e FITC (BioLegend, San Diego, CA) and anti-FITC microbeads (Miltenyi Biotec, Auburn, CA). Tcell-depleted BMCs contained $\mathrm{CD}^{+}$cells of $<0.5 \%$ by flow cytometry.

Donor BMCs were freshly injected within 3 hours after preparation. Briefly, the uteri of gestational day 14 pregnant FVB/N mice were exposed through a vertical laparotomy. A $60 \mu \mathrm{m}$ glass micropipette with beveled tip was used to inject $5-10 \times 10^{6}$ T-cell-depleted BMCs in $5-10 \mu \mathrm{L}$ of PBS into the peritoneal cavities of all fetuses at a litter via transuterine approach. Then, the abdomen of the pregnant mice was closed in two layers with 5-0 silk. After operation, all mice were housed in an undisturbed room without bedding changes until the pups were 1 week old. Pups were weaned at 3 weeks of age.

2.3. Analyses of Chimerism and MHC-I Expression of Donor Leukocytes. Chimerism levels were examined at the age of 1 month old. Peripheral blood was sampled via tail veins and depleted of red cells using ACK lysing buffer. Cells were first incubated with anti-mouse Fc $\gamma$ II/Fc $\gamma$ III antibody (BioLegend) and then stained with anti-H-2 $\mathrm{K}^{\mathrm{q}}$ FITC (BioLegend) and anti-H-2 $\mathrm{K}^{\mathrm{b}} \mathrm{PE}$ (BioLegend). Chimerism levels were determined by flow cytometry after gating out dead cells by high propidium iodide staining. A negative control consisted of anti-H-2K $\mathrm{K}^{\mathrm{q}}$ FITC and mouse IgG2a PE (BioLegend) to define background staining. Recipients with first month peripheral chimerism of $>3 \%$ were collected to further validate tolerance by skin transplantation because they could be consistently rendered tolerant to donor skin [11].
As for the quantification of donor leukocyte's MHC-I expression, engrafted donor leukocytes were gated, and the fluorescence intensities (FIs, including mean, geographic mean, and median) of anti-H-2 $\mathrm{K}^{\mathrm{b}} \mathrm{PE}$ were measured. Wild-type C57BL/ 6 mice were used as controls.

2.4. Skin Transplantation. Under anesthesia, the recipient's back was shaved by clippers and disinfected by beta-iodine solution. Graft beds with intact panniculus carnosus were created by the scissors [12]. Skin transplants from tails were placed on the graft bed, fixed by stitches, covered by Vaseline gauze, and dressed by Band-Aid. After the removal of dressings on day 7, grafts were monitored daily. Skin tolerance was defined by donor skin engraftment with good hair growth for at least 4 months. Engrafted donor skin was subjected to histological examinations after hematoxylin-eosin staining in order to exclude the possibility of microscopic rejection.

2.5. mRNA Extraction from Skin Grafts of Tolerant Mice. The engrafted skin grafts were taken down from tolerant mice at indicated time points. Then, their cell constituents were homogenized by physical treatment using a tissue tearor in Trizol Reagent (Invitrogen, USA). mRNAs were extracted with RNeasy Mini Kit (Qiagen K.K., Tokyo, Japan) according to the instruction manual. The purity of RNA samples was evaluated using absorption of light at 260 and $280 \mathrm{~nm}$ (A260/280) by a ND-2000 spectrophotometer (Nanodrop Technology, USA). High-quality mRNA showed the $\mathrm{A} 260 / 280$ ratio of $\geq 1.97$.

2.6. Quantitative Real-Time PCR for MHC Expression of Skin Grafts. First-strand cDNA were reversely transcribed from mRNA templates of each skin sample in equal amount, using MMLV High Performance Reverse Transcriptase Kit with an oligo(dT) primer (EPICENTRE Biotechnologies, Madison, WI). Then, MHC-I related target gene expression was quantified by Bio-RAD iQ5 real-time PCR detection system, using Maxima SYBR Green/ROX qPCR Master Mix (2X) (Fermentas, Thermo Scientific). The specificity of realtime PCR reaction was further confirmed by melting curve analysis. Normalized values for target mRNA expression in each sample were calculated as the relative quantity of target gene divided by the relative quantity of glyceraldehyde-3phosphate dehydrogenase (GAPDH). All the gene primers were designed by online primer design tool of Primer 3 as shown in Table 1.

2.7. Statistical Analyses. The equality of means was examined by Student's $t$-test between two independent or paired-sample groups. Differences were regarded as significant in case of $P<$ 0.05 .

\section{Results}

3.1. Screening of MHC Gene Expression in Engrafted Donor Skins. We collected 13 mixed chimeras with first month donor cell (C57BL/6) levels of 6.05 28.18\%. All were readily rendered tolerant to donor skin for at least 4 months 
TABLE 1: Primers used for quantitative real-time PCR.

\begin{tabular}{lcc}
\hline Gene & Forward primer & Reverse primer \\
\hline GAPDH & TCACCACCATGGAGAAGGC & GCTAAGCAGTTGGTGGTGCA \\
$\mathrm{H}-2 \mathrm{~K} \alpha$ & TGGTGCTTGTCTCACTGACC & CCGTTCTTCAGCATTTGGAT \\
$\mathrm{H}-2 \mathrm{D} \alpha$ & ACATGGAGCTTGTGGAGACC & TGTTGGAGACAGTGGATGGA \\
$\mathrm{H}-2 \mathrm{~L} \alpha$ & GCGGAGAATCCGAGATATGA & AGCCAGACATCTGCTGGAGT \\
$\mathrm{H}-2 \mathrm{~A} \alpha$ & GGAAAAGGAGGGGACTATGC & CAAGCTCACAGGGAACATCA \\
$\mathrm{H}-2 \mathrm{~A} \beta$ & GAGTCACACCCTGGAAAGGA & ACAGCCTCAGGGTCAAGAGA \\
Calnexin & GGGTCTCATCCACACAGCTT & ACATTTTGCTCCAGGCAGAC \\
Calreticulin & GGCTAGACGACGAACCTGAG & AGGCTTCCATTTGCCCTTAT \\
ERp57 & GACTTTCTGCCACCCAAGAA & TCCCACTCTCCATCCATCTC \\
TAP1 & TATGATGGGCCTAGGACTGC & TGCTGGCTGCTTTTAGGAAT \\
TAP2 & CATCACATCTCGGGTGACTG & TGCACTTTTCCCAGCTTCTT \\
Tapasin & GCTCCCTTTCAATGCCAATA & CACTGCATCCTGGATCTCCT \\
\hline
\end{tabular}

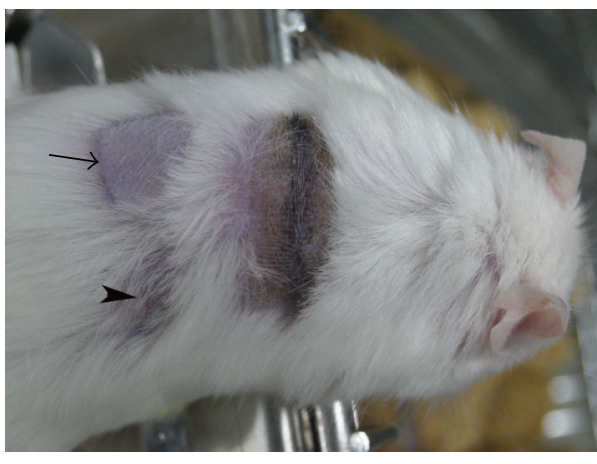

(a)

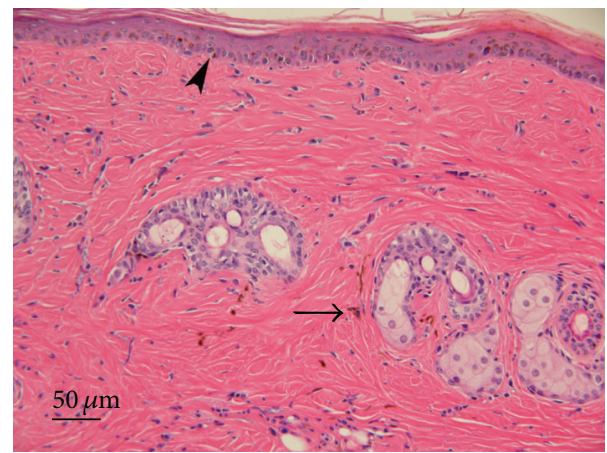

(b)

Figure 1: Donor-specific skin tolerance. Mixed chimerism was established in FVB/N mice through in utero transplantation of C57BL/6 marrows. A representative FVB/N mixed chimera was rendered tolerant to C57BL/6 donor skin (a). The donor-specific (black hair) and syngeneic (arrow) skins had been accepted for more than 4 months after their placement. The rejection of third-party $\mathrm{C} 3 \mathrm{H}$ skin caused an area of scarring (arrowhead). Hematoxylin-eosin staining did not reveal inflammatory cell infiltration in the engrafted C57BL/6 donor skin (b), which contained melanin pigments within keratinocytes of basal epidermis (arrowhead) and in the dermis (arrow).

(Figure 1). Engrafted donor skins had no histological evidence of inflammatory cell infiltration (Figure 1). Following mRNA extraction, 10 samples showed high-quality mRNA with $A 260 / 280$ of $\geq 1.97$. The remaining 3 samples were disqualified due to a lower A260/280 ratio of 1.84 1.92. Extracted mRNA from 5 wild-type C57BL/6 skins had the A260/280 ratio of $\geq 1.97$. Quantitative real-time PCR was then performed following reverse transcription from mRNA of each sample in equal amount. The mRNA expression of $\beta 2 \mathrm{~m}, \mathrm{H}-2 \mathrm{~K} \alpha, \mathrm{H}-2 \mathrm{D} \alpha, \mathrm{H}-2 \mathrm{~L} \alpha, \mathrm{H}-2 \mathrm{~A} \alpha$, and $\mathrm{H}-2 \mathrm{~A} \beta$ genes was determined by normalized fold expression relative to the mRNA quantity of GAPDH internal control gene. This survey for MHC gene expression revealed that engrafted donor skins significantly upregulated $\beta 2 \mathrm{~m}, \mathrm{H}-2 \mathrm{~K} \alpha, \mathrm{H}-2 \mathrm{D} \alpha$, and $\mathrm{H}-2 \mathrm{~L} \alpha$ as opposed to wild-type controls (Figure 2).

3.2. Gene Expression of Accessory Proteins in MHC-I Processing Pathway. We further examined the expression of mRNA that translates accessory proteins responsible for assembly and transport of MHC-I molecules. These chaperone-like proteins in MHC-I processing pathway include the transporter associated with antigen processing (TAP1 \& TAP2), tapasin, calnexin, calreticulin, and ERp57. Engrafted donor skins showed significant upregulation of TAP1, but only marginal upregulation of TAP2 (Figure 3).

3.3. MHC-I and TAP Gene Expression in Donor Skins before and after Transplantation. For further validating the upregulation of MHC-I related gene transcripts within tolerized grafts, we performed the matched-pairs studies for MHCI $(\beta 2 \mathrm{~m}, \mathrm{H}-2 \mathrm{~K} \alpha, \mathrm{H}-2 \mathrm{D} \alpha$, and $\mathrm{H}-2 \mathrm{~L} \alpha)$ and TAP1\&2 gene expressions of skin grafts before and after their transplantation. The mice with skin tolerance were used as recipients for secondary skin transplantation after removal of prior engrafted skins. Both allogeneic (C57BL/6) and syngeneic $(\mathrm{FVB} / \mathrm{N})$ tail skins were first collected before their placement on tolerant mice. Following skin transplantation, the engrafted skin graft from the same donor tail was harvested at indicated time points (1 2 weeks and $>1$ month). Following mRNA extraction $($ A260/280 ratio $\geq 1.97)$ and reverse 

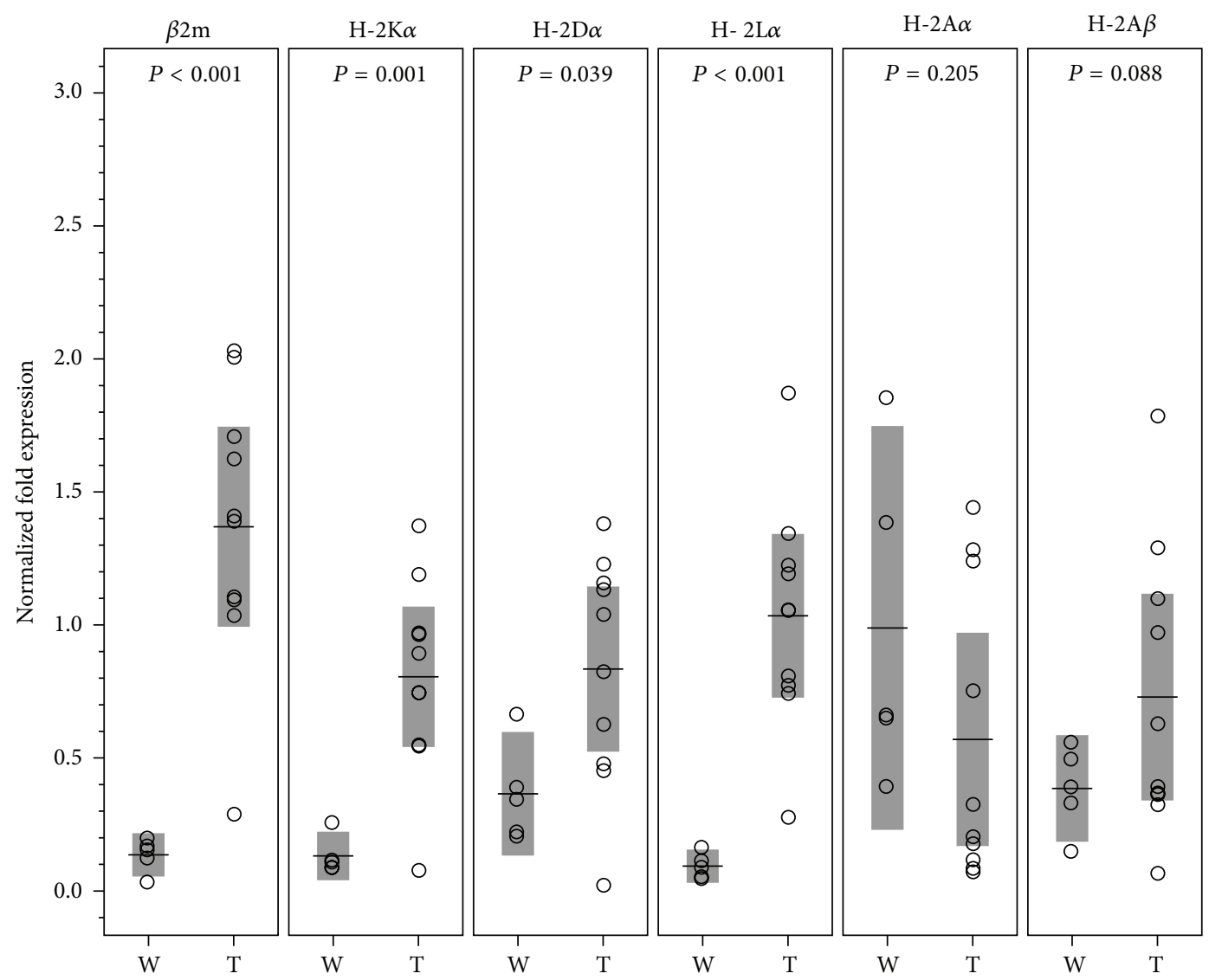

Figure 2: The expression of MHC related genes within engrafted C57BL/6 donor skins. MHC-I genes including $\beta 2 \mathrm{~m}, \mathrm{H}-2 \mathrm{~K} \alpha$, H-2D $\alpha$, and $\mathrm{H}-2 \mathrm{~L} \alpha$ were upregulated in engrafted donor skins (T) as compared with wild-type donor skins (W). However, the expressions of MHC class II (MHC-II) $\mathrm{H}-2 \mathrm{~A} \alpha$ and $\mathrm{H}-2 \mathrm{~A} \beta$ in engrafted and wild-type C57BL/6 skins were comparable. MHC-II H-2E is nonfunctional in C57BL/6 mice and not tested. The means and their $95 \%$ confidence intervals are shown as horizontal lines and shade boxes, respectively.

transcription, the expressions of target genes were measured in relation to those of an endogenous GAPDH reference gene by quantitative real-time PCR. Paired-sample $t$-test was used to compute the differences between values of the two normalized expression variables for each skin graft from the same donor before and after skin transplantation (pre-ST and post-ST). The expressions of $\beta 2 \mathrm{~m}, \mathrm{H}-2 \mathrm{~K} \alpha, \mathrm{H}-2 \mathrm{~L} \alpha$, and TAP1 were significantly increased in donor skins after shortterm (1 2 weeks) and long-term (>1 month) engraftment. However, $\mathrm{H}-2 \mathrm{D} \alpha$ was only significantly upregulated in shortterm engrafted donor skin (Figures 4 and 5). Syngeneic skin grafts did not exhibit significant upregulation of MHC-I related genes after engraftment.

3.4. Surface MHC-I Expression of Donor Leukocytes in Tolerant Mixed Chimeras. Surface MHC-I protein expression on engrafted donor leukocytes was quantified by flow cytometry. Five tolerant mixed chimeras with donor cell levels of 4.28 $35.12 \%$ at their age of 6 months were collected for the evaluation of $\mathrm{H}-2 \mathrm{~K}^{\mathrm{b}}$ mean, geographic mean, and median FIs. In tolerant mice, engrafted donor leukocytes had an about 3 4-fold increment of FIs as opposed to wild-type leukocytes (Figure 6).

\section{Discussion}

The first challenge that researchers face on initiating tolerance biomarker studies lies in the recruitment of subjects with operational tolerance $[2,13]$. Human recipients can be sometimes rendered tolerant to transplanted kidneys $[5,8]$ or livers [6]. In renal recipients, either gradual or abrupt withdrawal of immunosuppressive regimens may trigger episodes of acute rejection. This inevitably causes certain degree of irreversible renal parenchymal damage to jeopardize the function and longevity of renal allografts [1]. Although long-term sequelae from liver graft rejection may be minimal or even absent due to strong liver regenerative capacity, it is viable only when liver rejection episodes can be detected and treated early after immunosuppressive minimization or discontinuation [1]. Thus, it is not advisable to intentionally taper or discontinue immunosuppressive regimens in recipients for scrutinizing an existing state of operational tolerance.

Clinically, operational tolerance is usually a serendipitous clinical event, occurring in $10-20 \%$ of renal [14] and liver [15] recipients. This small fraction of recipients were usually recognized under a special situation wherein they did well after immunosuppressive discontinuation as a result of their 


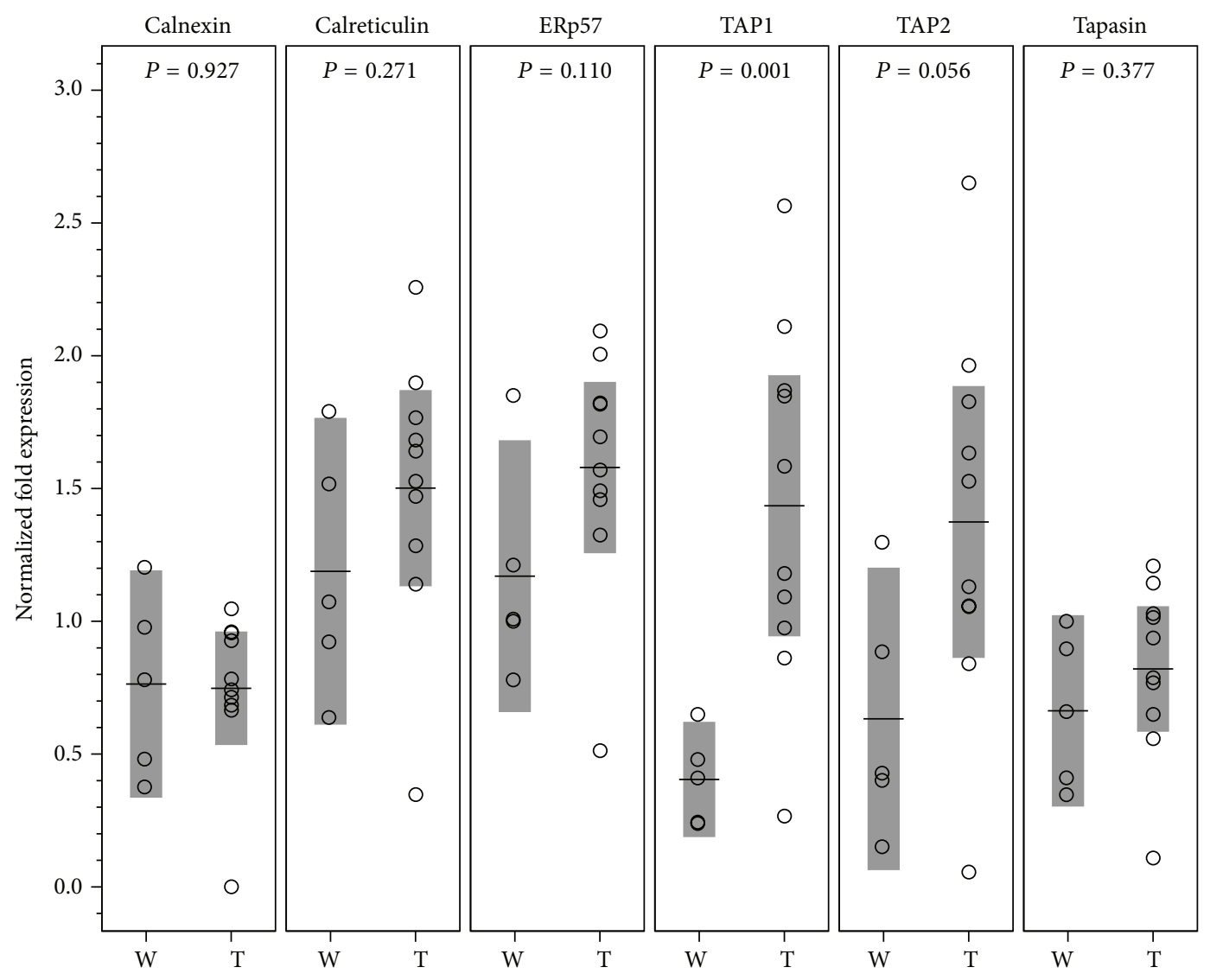

Figure 3: The mRNA expressions of accessory proteins in MHC-I pathway. As compared with wild-type donor skins (W), engrafted donor skins $(\mathrm{T})$ had significant upregulation of TAP1 $(P=0.001)$, but only borderline upregulation of TAP2 $(P=0.056)$. The means and their $95 \%$ confidence intervals are shown as horizontal lines and shade boxes, respectively.

medical noncompliance, or clinical necessity such as lifethreatening infection, malignancy, or unacceptable drug toxicity [1]. With the advent of high throughput biotechnology [16], these fortuitous recipients had been collected to profile their gene or proteome expression patterns [17]. The profiling of gene or proteome expressions requires appropriate controls for smoothing comparison so that researchers can identify specific variations attributed to a state or biological process of operational tolerance. However, operationally tolerant recipients were highly selected cases that had been universally subjected to long-term immunosuppressive therapies with diverse and complicated clinical courses. This made it difficult or even impossible to find appropriate control groups. In their simplest form, researchers may find the biomarkers that suffice to discriminate tolerance from nontolerance recipients. However, it is hard to ensure that the biomarkers are not merely a test to identify tolerance patients who are not taking immunosuppressive regimens [8]. Thus, it is important to enroll healthy cases given immunosuppressive regimens with and without subsequent immunosuppressive discontinuation so that we can analyze the contribution of immunosuppressive drugs or transplants to the profiles of assay results. However, it is merely a scientifically but not ethically sound approach. Despite this insurmountable obstacle in human subject studies for tolerance biomarkers, several studies had related operational tolerance to the alterations of natural killer (NK), regulatory (Treg), B- or $\gamma \delta$ T-cell associated gene expressions $[4,8,18]$.

The discovery of trivial donor leukocytes in the tissues or blood of long-surviving organ recipients had once led to the notion that graft tolerance was linked by a common dependence on the presence of donor cell chimerism $[19,20]$. It raised the expectations for using donor cell chimerism as an ideal tolerance biomarker. However, subsequent animal $[21,22]$ and human [23] studies showed that graft tolerance could persist in the absence of donor cell chimerism. In addition, graft rejection even occurred in a state of donor cell chimerism [24, 25]. This conflict might be ascribed to the emerging consensus that hematopoietic chimerism was relevant to the induction rather than the maintenance phase of graft tolerance [11, 26]. Despite the unreliability of donor cell chimerism as a tolerance biomarker [27, 28], the creation of mixed chimerism remains the most popular strategy to facilitate allograft tolerance in various animal models for the investigation of immune tolerance $[29,30]$. Surprisingly, there are few such animal studies seeking to identify tolerance biomarkers [1]. It is likely due to the notion that the results obtained in animal studies may not always apply to humans. However, animal studies remain the important touchstones of many biological phenomena in humans. 

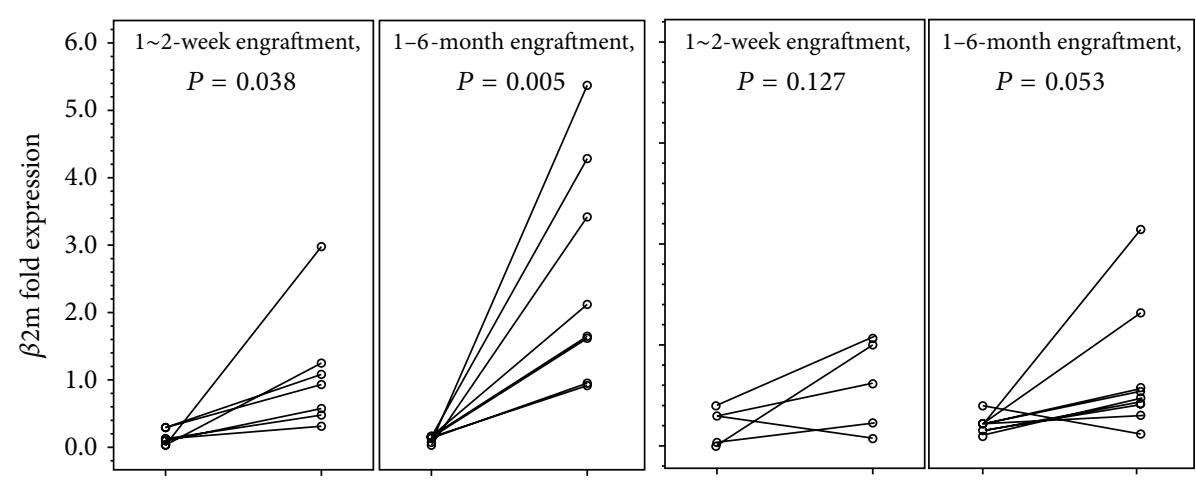

(a)
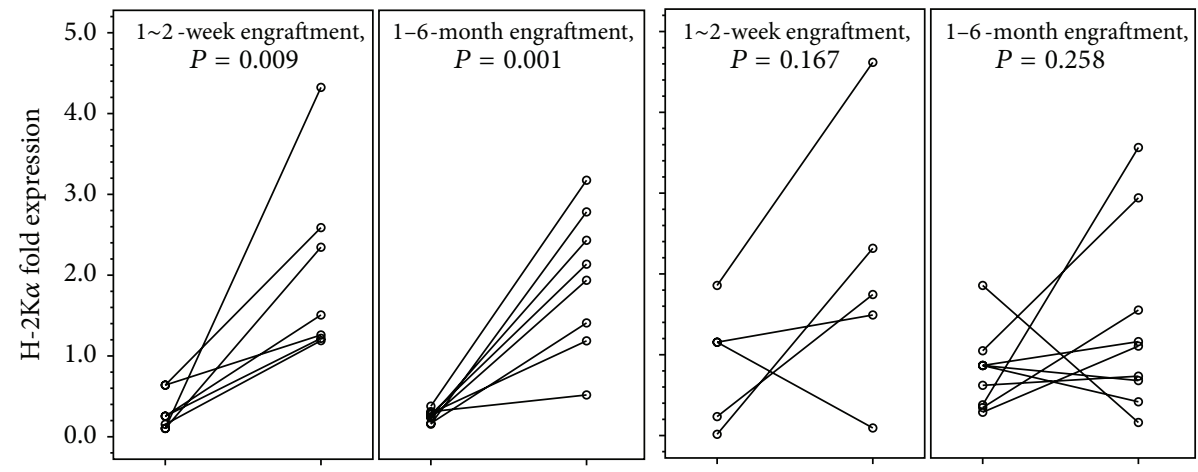

(b)
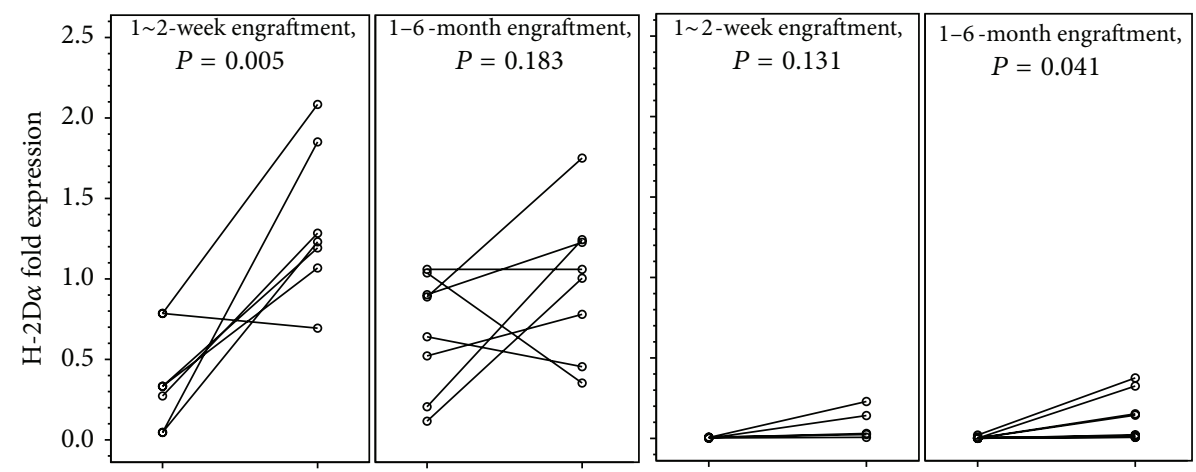

(c)
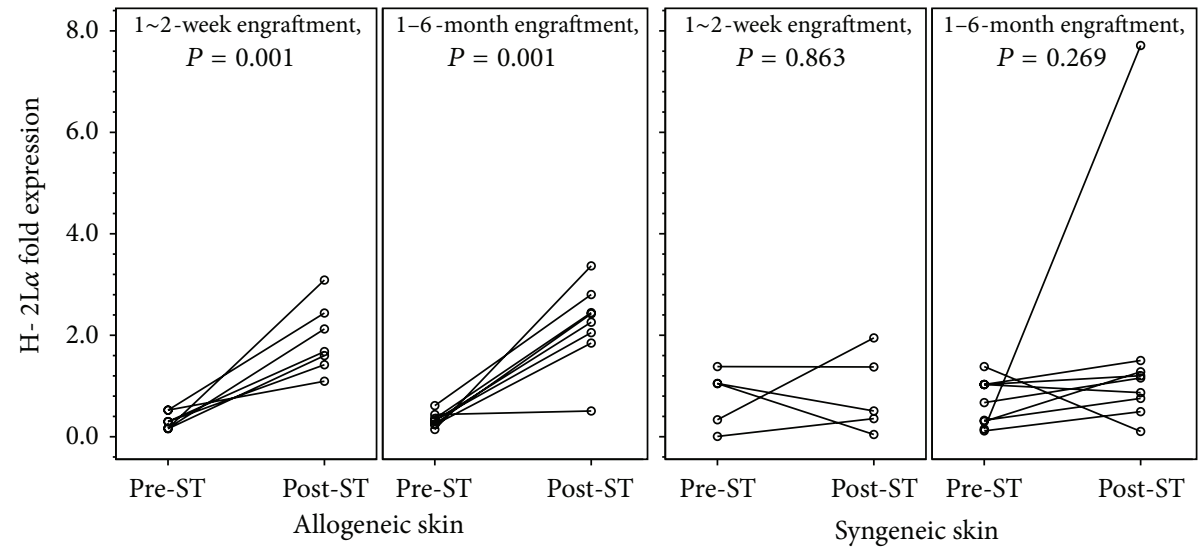

(d)

FIGURE 4: MHC-I upregulation in the process of donor skin tolerization. MHC-I related gene expression of donor skins was examined before (Pre-ST) and after (Post-ST) skin transplantation. The upregulation of $\beta 2 \mathrm{~m}(\mathrm{a}), \mathrm{H}-2 \mathrm{~K} \alpha(\mathrm{b}), \mathrm{H}-2 \mathrm{D} \alpha(\mathrm{c})$, and H-2L $\alpha$ (d) in donor skins showed up at the beginning of engraftment (1 2 weeks, $n=7)$ in tolerant mice and remained so within $1 \sim 6$ months $(n=8)$ after skin transplantation except for H-2D $\alpha$. Syngeneic donor skins did not exhibit the similar upregulation of MHC-I related genes after short-term $(1 \sim 2$ weeks, $n=5)$ and long-term (1 6 months, $n=9)$ engraftment. 

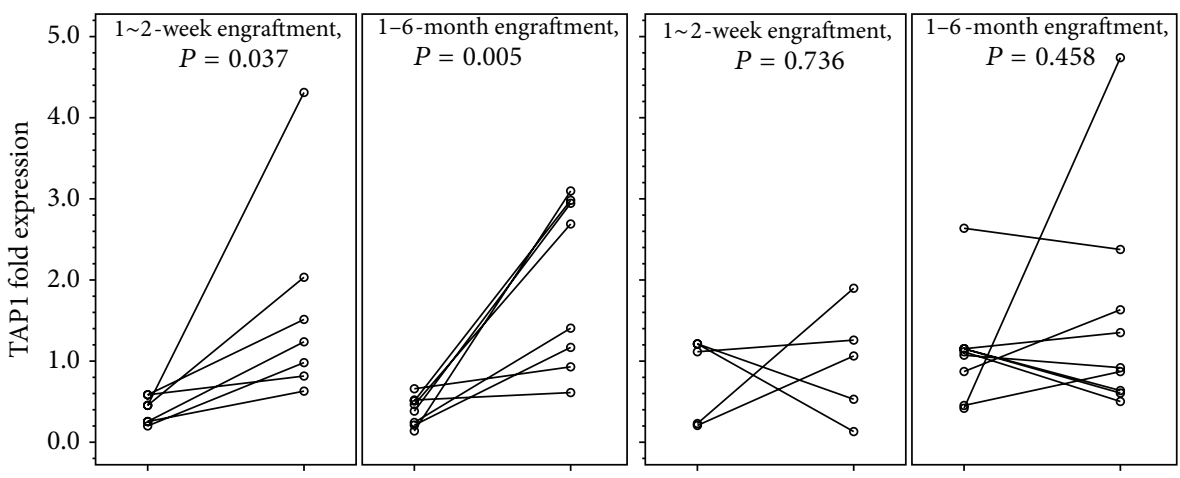

(a)

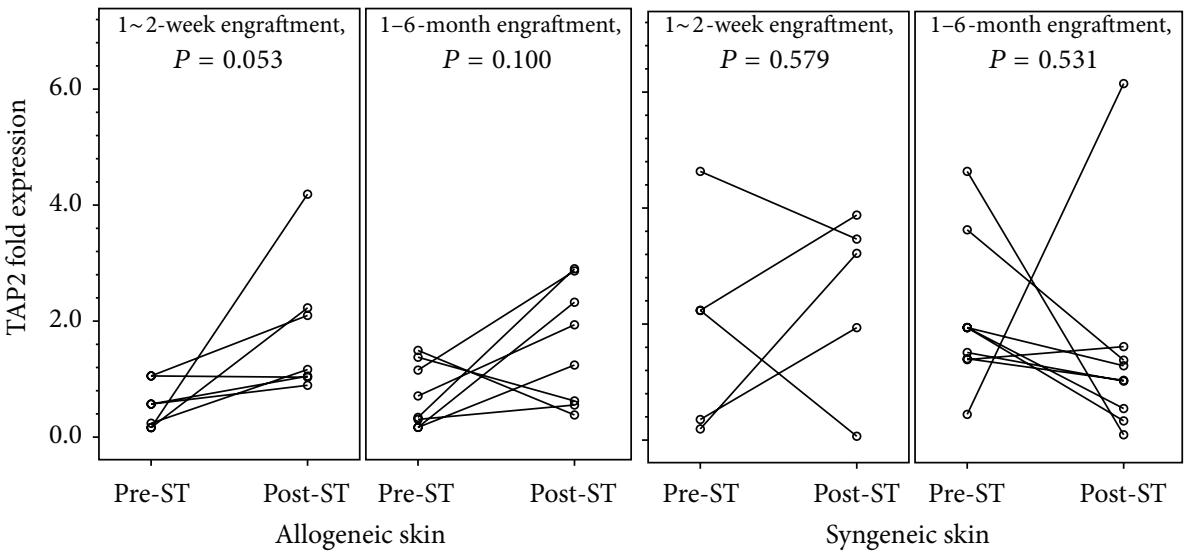

(b)

FIGURE 5: TAP1 and TAP2 expression in the process of donor skin tolerization. The engraftment of donor skins in tolerant recipients, either short-term $(n=7)$ or long-term $(n=8)$, enhanced the expression of TAP1 (a) rather than TAP2 (b) within donor skins. Neither TAP1 nor TAP2 was consistently upregulated within syngeneic skins after short-term $(n=5)$ and long-term $(n=9)$ engraftment.

The establishment of mixed chimerism to facilitate graft tolerance through conventional marrow transplantation necessitates myeloablative or immunosuppressive therapies to modify the host immunity [20,31]. Under the circumstances, tolerance biomarker studies still have to confront the interference of immunosuppressive therapies. In this regard, in utero marrow transplantation that requires no myeloablation and immunosuppression has unsurpassed advantage over conventional approach for tolerance-related studies. Thus, upregulation of MHC-I within engrafted transplants represented a biological phenomenon that was independent of immunosuppressive effects on the recipients.

In the pursuit of tolerance biomarkers, researchers mostly focused on the recipients in preference to the donor grafts for examining various biological activities or products that sufficed to signify a tolerant state. However, local regulatory mechanisms might contribute to graft tolerance due to Treg activity specifically within tolerated grafts $[32,33]$. Evidence also showed that Treg-associated gene expressions in recipients destined for graft tolerance and rejection primarily differed within the transplanted grafts themselves rather than systemically or in spleens and draining lymph nodes of recipients [18]. As a result, graft tolerance might not necessarily elicit a unique biomarker circulating systemically but rather be an immunological event occurring locally within the target transplants. Thus, it makes sense to search for tolerance biomarkers in tolerated grafts or cells despite the fact that biomarkers are preferably measurable in readily accessible sources such as hosts' blood. Allogeneic skins engrafted either short-term or long-term exhibited upregulation of MHC-I associated transcripts as opposed to syngeneic skin grafts. Thus, MHC-I upregulation did not result from the procedure of skin transplantation itself, but rather the biological modifications of donor allografts in the process of graft tolerance. A simultaneous upregulation of TAP1 gene transcripts suggested a kinetically critical role of TAP1 in the regulation of MHC-I pathway. MHC-I protein expression was quantified on engrafted donor leukocytes by flow cytometry. Engrafted donor cells consistently overexpressed surface $\mathrm{H}-2 \mathrm{~K}^{\mathrm{b}}$ (MHC-I).

Discovering a biomarker for transplantation tolerance is not necessarily equivalent to uncovering their causal relationship. However, our approach to the secondary donor skin acceptance in tolerant recipients suggested that MHC-I overexpression was the recipients' tolerizing effects on donor grafts. Within the donor grafts, inflammatory responses might upregulate their MHC-I and related genes. Graft inflammation usually resulted from alloimmune reactions. However, the absence of mononuclear cell infiltration reflected the lack of any ongoing inflammatory process 


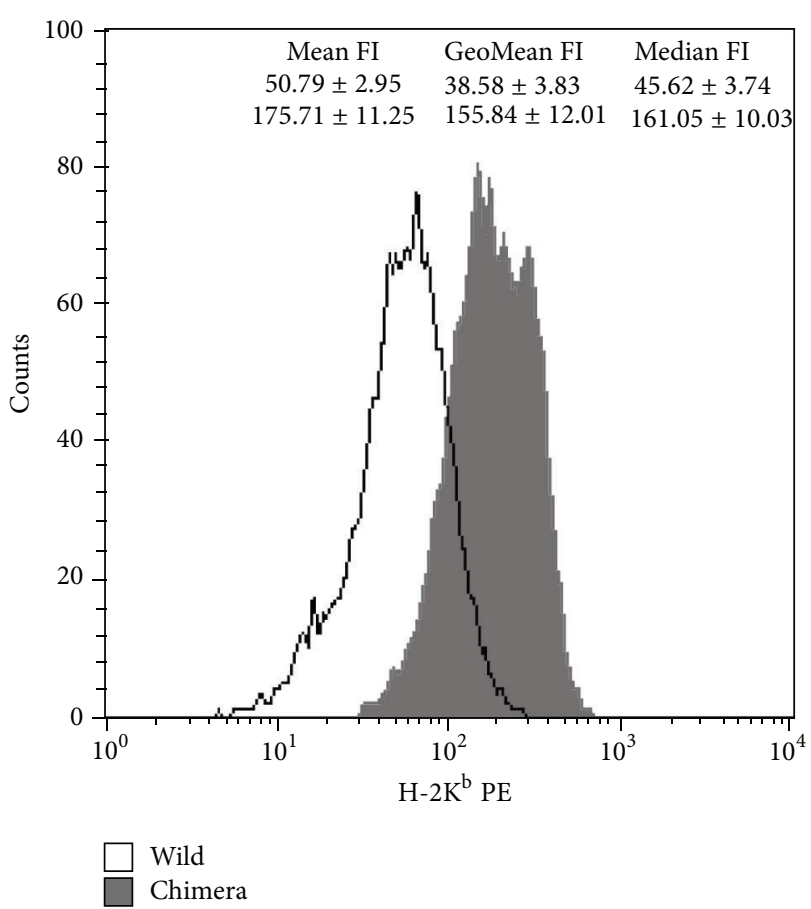

FIGURE 6: Surface H-2K $\mathrm{K}^{\mathrm{b}}$ FIs of engrafted donor leukocytes in 6month-old mixed chimeras with skin tolerance. This representative histogram showed surface $\mathrm{H}-2 \mathrm{~K}^{\mathrm{b}}$ PE FIs of C57BL/6 donor leukocytes from a mixed chimera and an age-matched wild-type C57BL/6 control. Chimeras $(n=5)$ had higher mean, geographic mean, and median $\mathrm{H}-2 \mathrm{~K}^{\mathrm{b}}$ FIs than wild-type C57BL/6 controls $(n=5)$. Data were expressed as mean \pm standard error of mean. $P<0.001$ for mean, geographic mean (GeoMean), and median FIs.

within the engrafted donor skins. Thus, MHC-I upregulation should be specific to tolerance rather than the results of alloimmunity. We have known that the alterations in MHCI expression play a crucial step in immune evasion of cancer or virus-infected cells through the regulation of adaptive Tcell cytotoxicity [34] and innate NK cell function [35]. Most cancer [9] or virus-infected cells [10] downregulate MHCI to escape adaptive T-cell cytolysis. In contrast, multidrugresistant human cancer cell lines and flaviviruses such as hepatitis C virus may upregulate MHC-I in parallel with TAP1 to evade innate NK cell attacks for the establishment of multidrug resistance [36] and chronic hepatitis $\mathrm{C}$ infection [37]. Given the critical role of NK cells in the defense against virus-infected, neoplastic, and allogeneic cells [38], the inhibition of NK cell cytotoxicity from the interaction of MHC-I ligands and NK inhibitory receptors [39, 40] may be related to the establishment of graft tolerance and awaits further experimental elucidation in the future.

\section{Conclusions}

Immune tolerance to donor transplants involved biological modifications of MHC-I upregulation within engrafted donor grafts. This biological phenomenon has great potential to be a tolerance biomarker in the settings of transplantation.
More importantly, it may pave the way to further mechanism studies for transplantation tolerance in the future.

\section{Conflict of Interests}

The authors declare that there is no conflict of interests regarding the publication of this paper.

\section{Acknowledgments}

This work was supported by the Grants NSC100-2314-B182A-043 and NSC 101-2314-B-182-093 (Jeng-Chang Chen) from National Science Council, Taiwan, and CMRPG4A0131 (Jeng-Chang Chen) from Chang Gung Memorial Hospital, Taiwan.

\section{References}

[1] L. A. Turka and R. I. Lechler, "Towards the identification of biomarkers of transplantation tolerance," Nature Reviews Immunology, vol. 9, no. 7, pp. 521-526, 2009.

[2] M. P. Hernandez-Fuentes and R. I. Lechler, "A "biomarker signature" for tolerance in transplantation," Nature Reviews Nephrology, vol. 6, no. 10, pp. 606-613, 2010.

[3] B. Sawitzki, P. Reinke, A. Pascher, and H. Volk, "State of the art on the research for biomarkers allowing individual, tailormade minimization of immunosuppression," Current Opinion in Organ Transplantation, vol. 15, no. 6, pp. 691-696, 2010.

[4] M.C-. Londoño, R. Danger, M. Giral, J.P-. Soulillou, A. SánchezFueyo, and S. Brouard, "A need for biomarkers of operational tolerance in liver and kidney transplantation," The American Journal of Transplantation, vol. 12, no. 6, pp. 1370-1377, 2012.

[5] S. Brouard, E. Mansfield, C. Braud et al., "Identification of a peripheral blood transcriptional biomarker panel associated with operational renal allograft tolerance," Proceedings of the National Academy of Sciences of the United States of America, vol. 104, no. 39, pp. 15448-15453, 2007.

[6] M. Martínez-Llordella, J. J. Lozano, I. Puig-Pey et al., "Using transcriptional profiling to develop a diagnostic test of operational tolerance in liver transplant recipients," The Journal of Clinical Investigation, vol. 118, no. 8, pp. 2845-2857, 2008.

[7] T. K. Sigdel and M. M. Sarwal, "The proteogenomic path towards biomarker discovery," Pediatric Transplantation, vol. 12, no. 7, pp. 737-747, 2008.

[8] P. Sagoo, E. Perucha, B. Sawitzki et al., "Development of a cross-platform biomarker signature to detect renal transplant tolerance in humans," The Journal of Clinical Investigation, vol. 120 , no. 6, pp. 1848-1861, 2010.

[9] A. Garcia-Lora, I. Algarra, and F. Garrido, "MHC class I antigens, immune surveillance, and tumor immune escape," Journal of Cellular Physiology, vol. 195, no. 3, pp. 346-355, 2003.

[10] D. Tortorella, B. E. Gewurz, M. H. Furman, D. J. Schust, and H. L. Ploegh, "Viral subversion of the immune system," Annual Review of Immunology, vol. 18, pp. 861-926, 2000.

[11] J. Chen, M. Kuo, L. Ou et al., "Characterization of tolerance induction through prenatal marrow transplantation: the requirement for a threshold level of chimerism to establish rather than maintain postnatal skin tolerance," Cell Transplantation, vol. 19, no. 12, pp. 1609-1622, 2010. 
[12] J. Chen, L. Ou, H. Yu, H. Chang, P. Chang, and M. Kuo, "Allogeneic lymphocytes exerted graft-versus-host rather than tolerogenic effects on preimmune fetuses," Journal of Surgical Research, vol. 183, no. 1, pp. 405-411, 2013.

[13] G. Orlando, P. Hematti, and R. J. Stratta, "Clinical operational tolerance after renal transplantation: current status and future challenges," Annals of Surgery, vol. 252, no. 6, pp. 915-928, 2010.

[14] J. Ashton-Chess, M. Giral, S. Brouard, and J. P. Soulillou, "Spontaneous operational tolerance after immunosuppressive drug withdrawal in clinical renal allotransplantation," Transplantation, vol. 84, no. 10, pp. 1215-1219, 2007.

[15] J. Lerut and A. Sanchez-Fueyo, "An appraisal of tolerance in liver transplantation," The American Journal of Transplantation, vol. 6, no. 8, pp. 1774-1780, 2006.

[16] P. A. Keown, W. R. McMaster, and B. M. McManus, "Tools to identify organ rejection and immune quiescence for biological understanding and personalized medical care," Biomarkers in Medicine, vol. 4, no. 1, pp. 115-121, 2010.

[17] O. Bestard, J. M. Cruzado, M. L. Franquesa, and J. M. Grinyó, "Biomarkers in renal transplantation," Current Opinion in Organ Transplantation, vol. 15, no. 4, pp. 467-473, 2010.

[18] S. P. Cobbold, E. Adams, and H. Waldmann, "Biomarkers of transplantation tolerance: more hopeful than helpful?" Frontiers in Immunology, vol. 2, article 9, 2011.

[19] T. E. Starzl, A. J. Demetris, N. Murase, S. Ildstad, C. Ricordi, and M. Trucco, "Cell migration, chimerism, and graft acceptance," Lancet, vol. 339, no. 8809, pp. 1579-1582, 1992.

[20] K. Wood and D. H. Sachs, "Chimerism and transplantation tolerance: cause and effect," Immunology Today, vol. 17, no. 12, pp. 584-587, 1996.

[21] T. Kawai, A. Poncelet, D. H. Sachs et al., "Long-term outcome and alloantibody production in a non-myeloablative region for induction of renal allograft tolerance," Transplantation, vol. 68, no. 11, pp. 1767-1775, 1999.

[22] T. Kawai, A. B. Cosimi, R. B. Colvin et al., "Mixed allogeneic chimerism and renal allograft tolerance in cynomolgus monkeys," Transplantation, vol. 59, no. 2, pp. 256-262, 1995.

[23] T. R. Spitzer, F. Delmonico, N. Tolkoff-Rubin et al., "Combined histocompatibility leukocyte antigen-matched donor bone marrow and renal transplantation for multiple myeloma with end stage renal disease: the induction of allograft tolerance through mixed lymphohematopoietic chimerism," Transplantation, vol. 68 , no. 4, pp. 480-484, 1999.

[24] H. J. Schlitt, J. Hundrieser, B. Ringe, and R. Pichlmayr, "Donortype microchimerism associated with graft rejection eight years after liver transplantation," The New England Journal of Medicine, vol. 330, no. 9, pp. 646-647, 1994.

[25] M. Hisanaga, J. Hundrieser, K. Böker et al., "Development, stability, and clinical correlations of allogeneic microchimerism after solid organ transplantation," Transplantation, vol. 61, no. 1, pp. 40-45, 1996.

[26] S. Ko, A. Deiwick, M. D. Jäger et al., "The functional relevance of passenger leukocytes and microchimerism for heart allograft acceptance in the rat," Nature Medicine, vol. 5, no. 11, pp. 12921297, 1999.

[27] A. P. Monaco and P. Medawar, "Chimerism in organ transplantation: conflicting experiments and clinical observations," Transplantation, vol. 75, no. 9, pp. S13-S16, 2003.

[28] S. H. Koretz, M. S. Gottlieb, S. Strober et al., "Organ transplantation in mongrel dogs using total lymphoid irradiation (TLI).," Transplantation Proceedings, vol. 13, no. 1, pp. 443-445, 1981.
[29] J. C. Chen, M. L. Chang, S. F. Huang et al., "Prenatal tolerance induction: relationship between cell dose, marrow T-cells, chimerism, and tolerance," Cell Transplantation, vol. 17, no. 5, pp. 495-506, 2008.

[30] T. Wekerle and M. Sykes, "Mixed chimerism as an approach for the induction of transplantation tolerance," Transplantation, vol. 68, no. 4, pp. 459-467, 1999.

[31] D. A. Hale, R. Gottschalk, A. Umemura, T. Maki, and A. P. Monaco, "Establishment of stable multilineage hematopoietic chimerism and donor-specific tolerance without irradiation," Transplantation, vol. 69, no. 7, pp. 1242-1251, 2000.

[32] S. P. Cobbold, R. Castejon, E. Adams et al., "Induction of foxP3+ regulatory $\mathrm{T}$ cells in the periphery of $\mathrm{T}$ cell receptor transgenic mice tolerized to transplants," Journal of Immunology, vol. 172, no. 10, pp. 6003-6010, 2004.

[33] L. Graca, S. P. Cobbold, and H. Waldmann, "Identification of regulatory T cells in tolerated allografts," Journal of Experimental Medicine, vol. 195, no. 12, pp. 1641-1646, 2002.

[34] A. R. Townsend, J. Rothbard, F. M. Gotch, G. Bahadur, D. Wraith, and A. J. McMichael, "The epitopes of influenza nucleoprotein recognized by cytotoxic $\mathrm{T}$ lymphocytes can be defined with short synthetic peptides," Cell, vol. 44, no. 6, pp. 959-968, 1986.

[35] H.-G. Ljunggren and K. Karre, "In search of the 'missing self': MHC molecules and NK cell recognition," Immunology Today, vol. 11, no. 7, pp. 237-244, 1990.

[36] M. A. Izquierdo, J. J. Neefjes, A. E. L. Mathari, M. J. Flens, G. L. Scheffer, and R. J. Scheper, "Overexpression of the ABC transporter TAP in multidrug-resistant human cancer cell lines," British Journal of Cancer, vol. 74, no. 12, pp. 1961-1967, 1996.

[37] K. Herzer, C. S. Falk, J. Encke et al., "Upregulation of major histocompatibility complex class I on liver cells by hepatitis C virus core protein via p53 and TAP1 impairs natural killer cell cytotoxicity," Journal of Virology, vol. 77, no. 15, pp. 8299-8309, 2003.

[38] F. Colucci, M. A. Caligiuri, and J. P. Di Santo, "What does it take to make a natural killer?" Nature Reviews Immunology, vol. 3, no. 5, pp. 413-425, 2003.

[39] E. O. Long, "Regulation of immune responses through inhibitory receptors," Annual Review of Immunology, vol. 17, pp. 875-904, 1999.

[40] L. L. Lanier, "NK cell receptors," Annual Review of Immunology, vol. 16, pp. 359-393, 1998. 


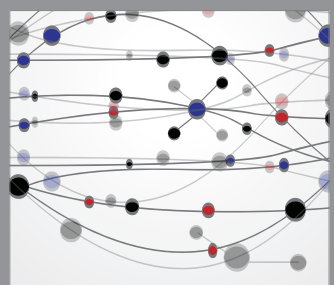

The Scientific World Journal
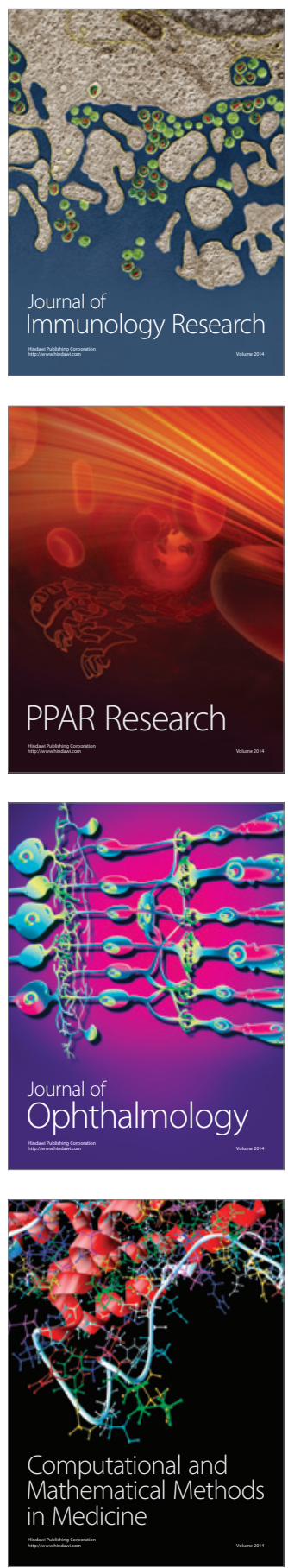

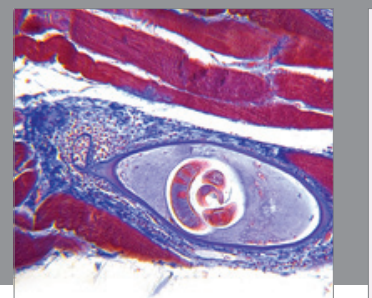

Gastroenterology

Research and Practice
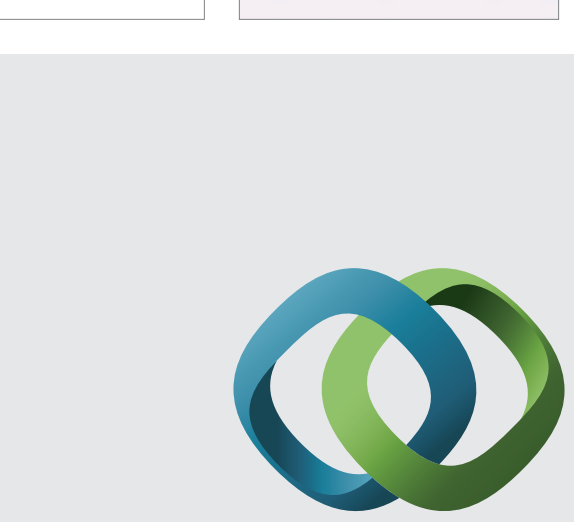

\section{Hindawi}

Submit your manuscripts at

http://www.hindawi.com
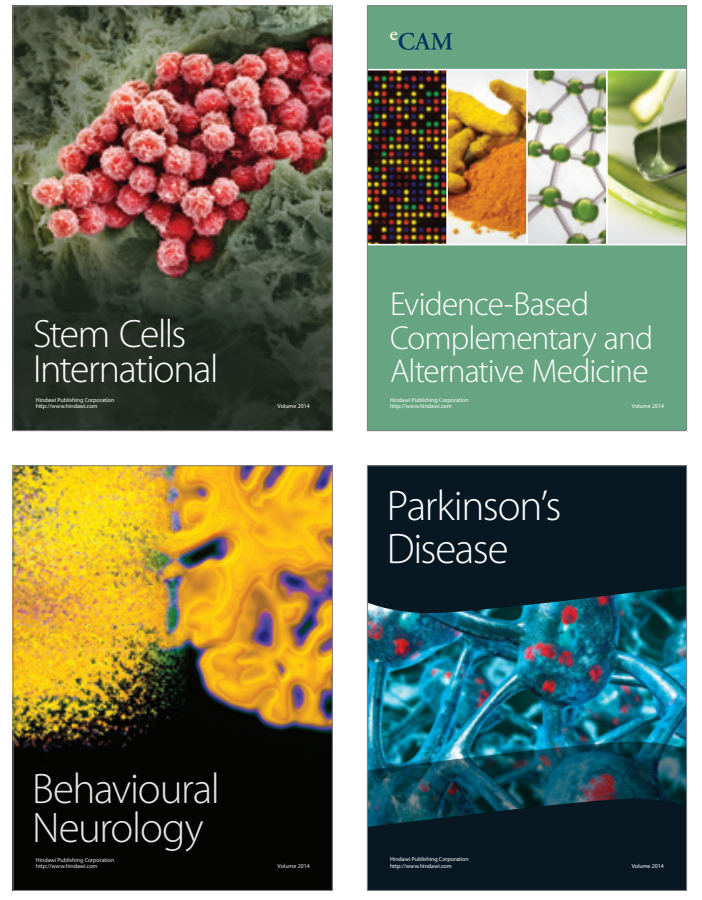
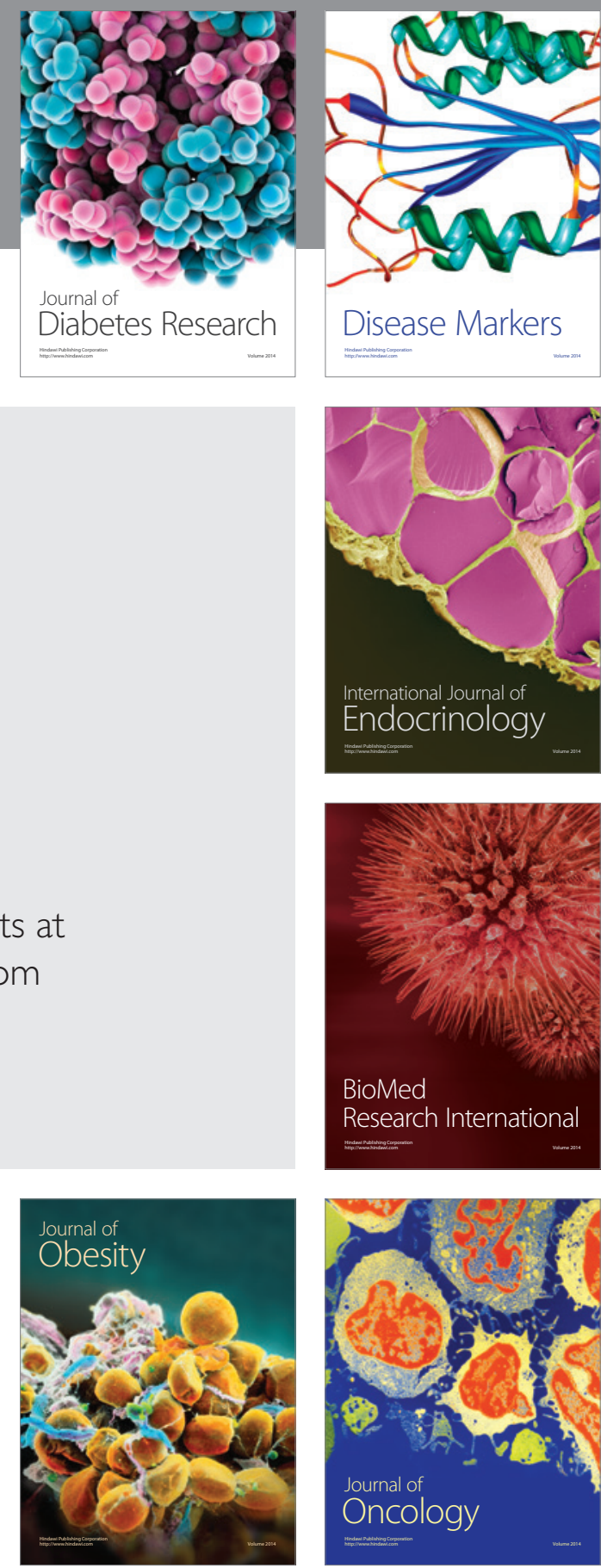

Disease Markers
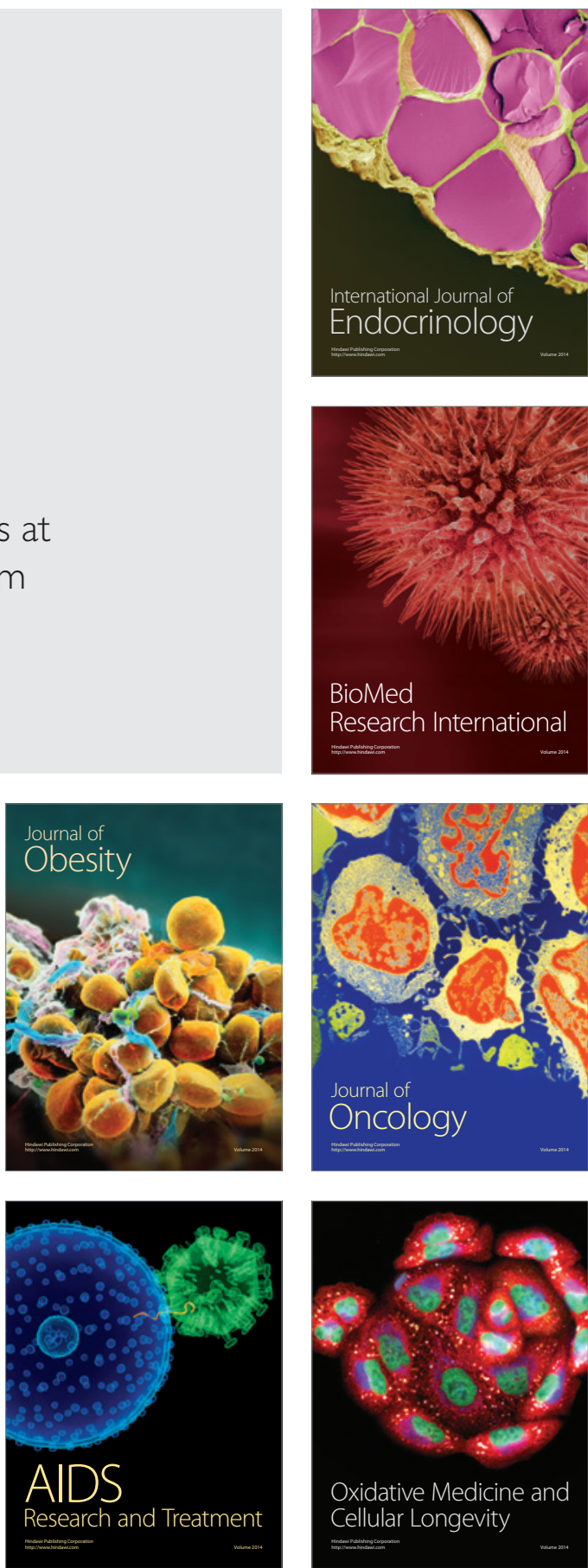